\title{
Editorial
}

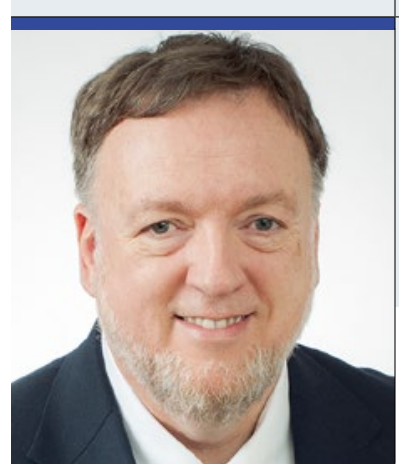

"Der Nachwuchs wird sich nur aus der Mitte der schmerz-

medizinisch versorgenden Kollegen generieren lassen -

dazu wird ein Funke der Begeisterung benötigt, der auf jüngere

Kollegen überspringen kann."

\section{Woher kommt der Nachwuchs?}

G lauben Sie ebenso wie viele Kollegen, dass das gefühlte Durchschnittsalter der praktizierenden Schmerzmediziner steigt? Und dass die Nachbesetzung von schmerzmedizinischen Sitzen in Praxis und Klinik gleichzeitig schwieriger wird? Es existieren zwar keine offiziellen Zahlen, aber im Dialog mit vielen Kollegen zeichnet sich ab, dass die schmerzmedizinische Versorgung in Deutschland auf den Schultern einer immer älter werdenden Ärztegeneration ruht. Gleichzeitig steigt nachweislich die Zahl der Patienten mit chronischen Schmerzen. Im Studentenunterricht erfahre ich, dass die Schmerzmedizin nur mit einem interessanten und schlüssigen Angebot für sich werben kann. Notwendig scheint mir, strategische Handlungsfelder zu definieren, in denen wir in den nächsten Jahren gemeinsam handeln sollten, um uns interessanter zu machen als bisher. Diese Felder sind: 1. Versorgungsstruktur: Wir brauchen in der ambulanten Medizin eine verlässliche Bedarfsplanung, in der sich perspektivisch ein Lebensplan oder eine Karriere realisieren lässt. Diese Verlässlichkeit schließt die angemessene Vergütung unseres ärztlichen Handelns ein. Inzwischen wissen wir allerdings, dass die Vergütung nicht vorrangig zählt, sondern die Attraktivität unseres beruflichen Alltags. Wir sollten daher die Verzahnung der Schmerzmedizin mit den anderen klinischen Fächern glaubwürdiger vorleben und konkreter vertraglich regeln.

2.Patientennähe: Der besondere Reiz schmerzmedizinischer Tätigkeit liegt in einem patientenorientierten weiten Kanon zwischen technischen, pharmakologischen, kommunikativen und psychosomatischen Kompetenzen.

3. Digitalisierung: Die junge Generation ist offener und ansprechbarer für alle Formen der Kommunikation und Vernetzung in digitaler Form.

4. Versorgungsforschung: Die jungen Kollegen sind es leid, die Glaubwürdigkeit und Relevanz der Xten Studie zu einem klinischen Problem zu prüfen oder gar Leitlinien, die von Autoren erstellt werden, die selbst nicht in der Patientenversorgung aktiv sind. Glaubwürdige Daten zur schmerzmedizinischen Realität müssen von uns Schmerzmedizinern selbst nachhaltig erzeugt und gepflegt werden (siehe iDocLive ${ }^{\circ}$.

5.Ausbildung: Über die universitären Lehrstühle kann schmerzmedizinischer Nachwuchs kaum gewonnen werden. Der Nachwuchs wird sich meiner Meinung nach nur aus der Mitte der schmerzmedizinisch versorgenden Kollegen generieren über Hospitation, Mitarbeit, Einbeziehung in Schmerzkonferenzen und positive Vertretung des Fachs Schmerzmedizin gegenüber den jungen Kollegen; dazu wird ein Funke der Begeisterung benötigt, der auf jüngere Kollegen überspringen kann.

6. Fort- und Weiterbildung: Dringend werden Angebote an jüngere Kollegen jenseits der mittlerweile universitär etablierten Curricula (Q 13) benötigt, um in der Hausarzt- und Facharztweiterbildung schmerzmedizinische Themen früh zu etablieren (und in den Facharztprüfungen auch zu prüfen).

7. Interdisziplinarität: Der chronische Schmerzpatient ist in der Regel ein multimorbider Patient, der die Zusammenarbeit mit allen beteiligten Fächern erfordert. Die jungen Kollegen erwarten flache Hierarchien, in denen sich die Beteiligten auf Augenhöhe begegnen.

8. Facharzt für Schmerzmedizin: auch als Ausbildungsperspektive für junge Ärzte.

9. Grundlagenforschung als l'art pour l'art? Hier sind Karriereoptionen auch für den Nachwuchs zu schaffen.

Schmerzmedizin kann nur dann für unseren Nachwuchs attraktiver werden, wenn wir mehr als bisher Anlass bieten, sich mit unserer Tätigkeit zu identifizieren. Daran sollten wir gemeinsam verstärkt arbeiten, denn Jammern hilft nicht.

Ihr

Johannes Horlemann 